\title{
Bovine gamma/delta $T$ cells are stimulated by bovine coronavirus antigens identified by a soluble $\mathrm{T}$ cells receptor*)
}

\author{
FELIX N. TOKA
}

\begin{abstract}
Department of Preclinical Sciences, Faculty of Veterinary Medicine, Warsaw University of Life Sciences, Ciszewskiego 8, 02-787 Warsaw, Poland and Ross University School of Veterinary Medicine, Basseterre, St. Kitts \& Nevis, West Indies
\end{abstract}

Felix N. Toka

\section{Bovine $\gamma \delta$ T cells are stimulated by bovine coronavirus antigens identified} by a soluble $\mathrm{T}$ cells receptor

\section{Summary}

Gamma/delta $(\gamma \delta) T$ cells in cattle account for an abundant $T$ cell population. However, little is known regarding the function of $\gamma \delta$ T cells as immune cells compared to $\alpha \beta$ T cells. Not many pathogen-related antigens have been defined and known to stimulate $\gamma \delta$ T cells. To address this information gap, we constructed a soluble receptor for bovine $\gamma \delta \mathrm{T}$ cells $(\mathrm{s} \gamma \delta \mathrm{TCR})$ that was later used to identify two proteins $(156 \mathrm{kDa}$ and $102 \mathrm{kDa})$ or protein fragments expressed by bovine coronavirus $(\mathrm{BCov})$. The molecular weight of the larger protein suggests it could be the spike glycoprotein of BCov. Subsequently, we used the identified viral proteins to study the reactivity of bovine $\gamma \delta$ T cells. In vitro assays showed that purified preparations of the two proteins stimulated $\mathrm{WC} 1+\gamma \delta \mathrm{\delta}$ cells isolated from cattle. A 4-fold increase in IFN $\gamma$ production and a significantly higher expression of MHC class II was observed. However, these viral ligands could not stimulate $\gamma \delta$ T cells to synthesize IL-8 or GM-CSF, known to be produced by $\gamma \delta \mathrm{T}$ cells when stimulated with bacterial antigens. Although the $\gamma \delta \mathrm{T}$ cells assessed here appeared activated by way of IFN $\gamma$ and MHC II expression, surface markers such as CD2, CD25, CD44, CD62L and CD335 were not expressed at significant levels. Further, the activation elicited by viral ligands was not sufficient to induce cytotoxic capability in $\gamma \delta \mathrm{T}$ cells in vitro as measured by a flow cytometrybased cytotoxicity assay. This in vitro study shows that $\mathrm{WC} 1+\gamma \delta \mathrm{T}$ cells can directly recognize viral antigen.

Keywords: gamma/delta $T$ cells, viral ligands, bovine coronavirus, soluble $\gamma \delta T C R$

Gamma/delta T lymphocytes ( $\gamma \delta \mathrm{T}$ cells) in young ruminants account for at least $35-60 \%$ of the total lymphocytes in blood circulation (15). This unique feature of ruminants is beneficial from the research stand point because it allows acquisition of enough cells from just a few milliliters of blood without overtly stressing animals. Although $\gamma \delta$ T cells are numerous in circulation, their role in immune response during infection is poorly understood. Moreover, the mechanisms involved in the activation of $\gamma \delta$ T cells are not clear. It is for these reasons that bovine $\gamma \delta$ T cells are of interest to veterinary immunologists as a research target. Bovine $\gamma \delta$ T cells are separated into two main groups based on the expression of the $\mathrm{WC} 1$ molecule (4). $\mathrm{WC}^{+} \gamma \delta \mathrm{T}$ cells are reported to play an inflammatory role (14), while those expressing $\mathrm{WC} 1.1^{+}$are suggested to be regulatory,

*) This work was supported by the Ministry of Science and Higher Education, Grant NN308566040 to FNT. although other associations have been made by others $(9,11)$. Maślanka et al. (10) associate the suppressor profile of $\gamma \delta$ T cells with expression of CD25, and WC1 with production of IFN $\gamma$, IL-10 and TGF-beta. WC1 ${ }^{+}$ $\gamma \delta \mathrm{T}$ cells have been reported to have a role in innate immune responses, e.g., they are capable of IFN $\gamma$ secretion and cytotoxicity against virus-infected cells, and are able to present antigen (18). Infection of bovine macrophages with Mycobacterium avium subspecies paratuberculosis can be modified by the presence of $\mathrm{WC}^{+} \gamma \delta \mathrm{T}$ cells (3). On the other hand, other infectious agents, such as low virulence or high virulence bovine viral diarrhoea virus, can induce depletion or redistribution of $\mathrm{WC}^{+} \gamma \delta \mathrm{T}$ cells upon infection, as reported by Palomares et al. (13). Knowledge about the nature of ligands recognized by $\gamma \delta$ T cells is just as unclear (8), but it is likely to contribute to the rational design of vaccines against many infectious agents of 
humans and animals. The immunomodulatory nature of some of the $\gamma \delta \mathrm{T}$ cell ligands has shown promise in treatment of various diseases $(7,12)$. Because antigen recognition by $\gamma \delta$ T cells is said to occur without the participation of major histocompatibility complex (MHC) class I and II molecules, a hypothesis was put forward that antigen recognition takes place directly. Therefore, the recognition of antigen may be similar to that observed for B lymphocytes. The aim of this study was to determine the nature of viral ligands recognized by $\gamma \delta \mathrm{T}$ cell receptor in cattle through reactivity with a soluble $\gamma \delta$ T cell receptor ( $s \gamma \delta \mathrm{TCR})$. For this purpose a s $\gamma \delta$ TCR was constructed using molecular biological techniques. Results showed that the soluble receptor could recognize viral antigens that were later used to stimulate $\mathrm{WC}^{+}$cells, evidenced by IFN $\gamma$ secretion and increase in $\mathrm{MHC}$ class II expression.

\section{Material and methods}

Peripheral blood mononuclear cells (PBMC). To prepare PBMCs, blood was collected from Polish lowland black and white cattle (abattoir animals) into heparin tubes. Cells were isolated by centrifuging blood mixed with PBS (1: 1 ratio) on Histopaque $(1.077 \mathrm{~g} / \mathrm{mL}$, Sigma-Aldrich, USA). Later, $\mathrm{WC}^{+}$cells were separated with MACS (Miltenyi Biotec, Germany) as described elsewhere (18). The purity of cells was usually $96-97 \%$ (data not shown). To proportionally increase various clones of $\mathrm{WC}^{+}$cells, cytokines such as IL-2 (R\&D Systems, USA), IL-12 (Kingfisher Biotech, Inc., MN USA), hIL-15 (R\&D Systems, USA) and hIL-18 (R\&D Systems,_USA) were used for stimulation for $48 \mathrm{~h}$. Isolation of different clones of $\mathrm{WC}^{+}$ cells was achieved through limiting dilution so that at least 1 to 4 cells were obtained and later expanded in the presence of cytokines that supported their initial proliferation.

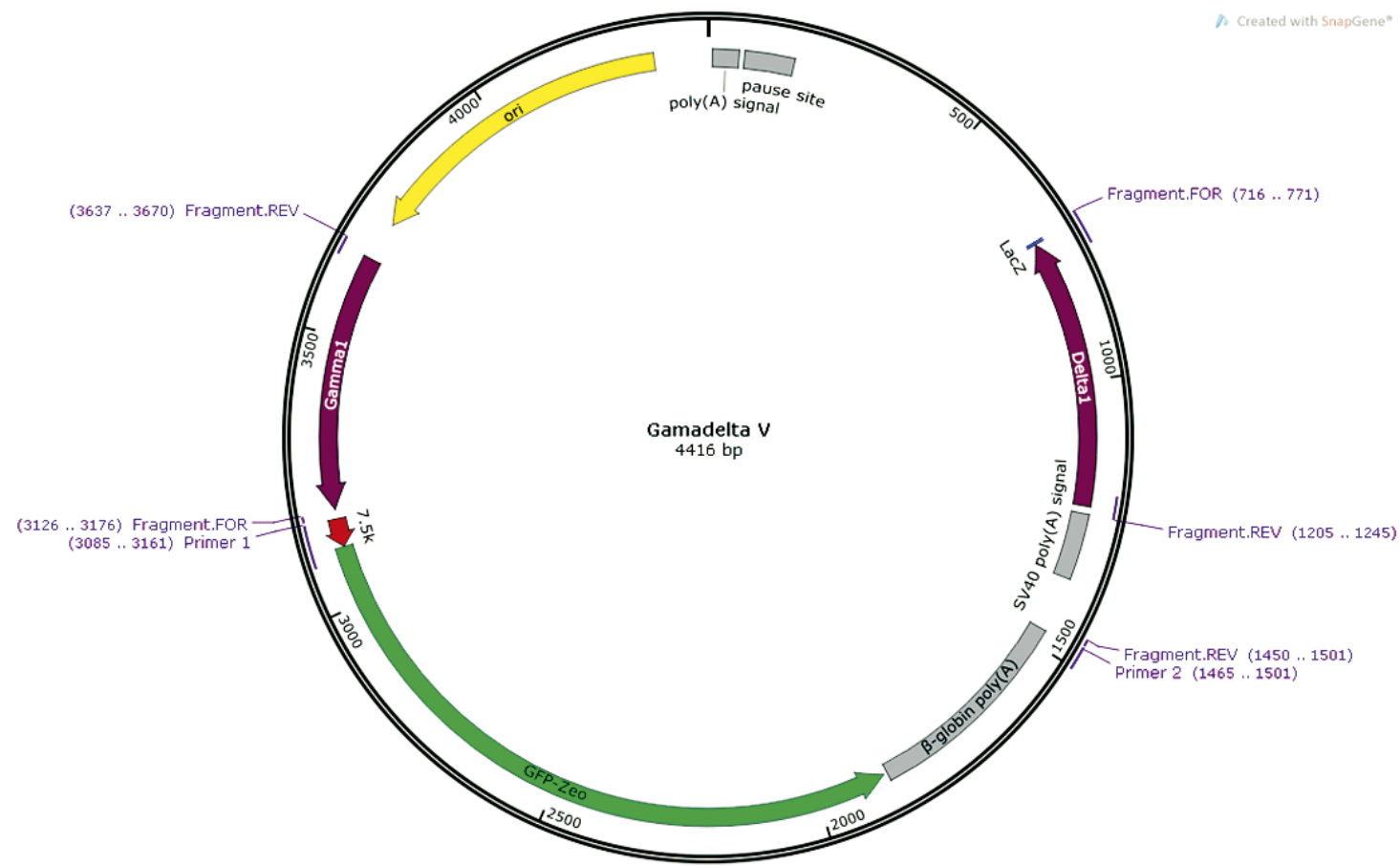

Fig. 1. Cloning strategy and baculovirus vector construction

Explanations: Vector planning and design were performed with Snapgene software.
Tab. 1. Primer names and sequences

\begin{tabular}{|l|l|}
\hline \multicolumn{1}{|c|}{ Primer name } & \multicolumn{1}{c|}{ Sequence (5' -3') $^{\prime}$} \\
\hline TDd1-for & GCAGATAAATCCATCAGCCTCC \\
\hline TDd2-for & CACAGAACTCCATCAGCCTCC \\
\hline TDd3-for & TCACGTAAAGCCATCAGCCTCATT \\
\hline TGg1-for & GGAAAAAAAATCATCAGCCTCACC \\
\hline TGg2-for & TACAAACCCAACCAAATGCTGAAA \\
\hline TGg3-for & AGCATGAGCCAAAAACCTTCCAC \\
\hline TDG-rev & CTCCTTCACCAAACAAGCGACG \\
\hline
\end{tabular}

Proliferation was assessed by the MTT test as described elsewhere (5), and optical densities were measured in an Epoch spectrophotometer (Biotek, USA). Expansion assay were carried out for $5-7$ days at $37^{\circ} \mathrm{C}, 5 \% \mathrm{CO}_{2}$ in RPMI-1640 GlutaMax (Life Technologies, ThermoFisher Scientific, USA) medium supplemented with $10 \%$ fetal bovine serum (Life Technologies, ThermoFisher Scientific, USA). To determine the diversification of the $\gamma \delta \mathrm{TCR}$, different clones were spectratyped on the basis of the CDR3 region by PCR and electrophoretic separation. Fluorochromelabelled primers (Sigma-Aldrich, MO, USA) to amplify $\gamma$ and $\delta$ CDR3 regions are shown in Tab. 1. Rneasy (Qiagen, USA) was used for RNA isolation and preamplification Superscript (Invitrogen, USA) was used for PCR. Amplification conditions were $95^{\circ} \mathrm{C}$ for $10 \mathrm{~min} ., 55^{\circ} \mathrm{C}$ for $1 \mathrm{~min}$., and $72^{\circ} \mathrm{C}$ for $1 \mathrm{~min}$., for denaturing, annealing and elongation, respectively for 40 cycles. Spectratype results were measured in Kodak In Vivo Imaging System (Bio-Rad, USA) using the fluorescence option and were used to select $\mathrm{WC}^{+} \gamma \delta$ T cells for $\gamma$ and $\delta$ chain cloning.

Cloning and expression of $\boldsymbol{\gamma}$ and $\boldsymbol{\delta}$ chains. RNA was isolated and transcribed to cDNA and later amplified by PCR using primers with inserted termination codons such that only the extra region of both chains were amplified, essentially as described in Aydintug et al. (2) and Davodeau et al. (6).

Amplicons were then cloned sequentially into pAcUW51 plasmid between EcorI and BamHI, XhoI and $B p u$ as depicted in Fig 1. Baculoviral vectors expressing s $\gamma \delta$ TCR were constructed by transfecting sf9 (Spodoptera frugiperda) cells with pAcUW51 containing $\gamma$ and $\delta$ chains together with baculovirus DNA sequences using the BaculoGold system (BD Biosciences, USA). Baculovirus produced in this manner was later used to infect BTI-TN-5B1-4 cells (Trichoplusia ni, Invitrogen) 
cultured in Express Five serum-free medium. Soluble $\gamma \delta$ TCR was detected in supernatants by ELISA with anti- $\gamma$ and anti- $\delta$ antibodies (VMRD. Inc., USA). Supernatants with s $\gamma \delta$ TCR were subjected to purification by affinity chromatography in columns containing sepharose with immobilized anti- $\gamma \delta$ antibodies and later eluted in a low pH buffer. Finally, Centricon filters (Millipore, USA) were used to concentrate the s $\gamma \delta T C R$.

Virus ligands. The bovine coronavirus $\left(\mathrm{ATCC}^{\circledR}\right.$ VR-874TM , BCov) was propagated in HRT-18 cells (COLO 201 (ATCC ${ }^{\circledR}$ CCL-224TM). Supernatants were clarified and concentrated, and later used in viral ligand detection. To assess the binding of s $\gamma \delta$ TCR to viral ligands, we adapted an immunoblotting technique, in which the viral lysates were electrophoresed and later transferred to a PVDF membrane, followed by incubation in a solution containing the $s \gamma \delta \mathrm{TCR}$. Bound complexes were detected with anti- $\gamma \delta$ antibodies. Viral proteins at band positions indicated in the immunoblotting detection were excised, eluted, concentrated in centricon filters and finally used to test for stimulatory properties on $\gamma \delta \mathrm{T}$ cells isolated from PBMCs.

Stimulation of $\gamma \delta$ T cells isolated from PBMCs. $\gamma \delta$ T cells were isolated as described earlier and incubated in the presence of eluted proteins p 102 or p 156 at $1 \times 10^{6}$ cells per well (in triplicates) in a 96-well plate. Cells without addition of eluted proteins were treated as non-stimulated (NS) and served as negative control, while the cells that served as positive control were treated with concanavalin $\mathrm{A}(5 \mu \mathrm{g} /$ $\mathrm{ml}$, Sigma-Aldrich, USA). For the assessment of cytokine synthesis, cells were incubated for 18-24 h in RPMI-1640 composed as mentioned earlier. At least $4 \mathrm{~h}$ before the end of incubation, brefeldin A (eBiosciences, USA) was added at a concentration of $3.0 \mathrm{ug} / \mathrm{ml}$. Intracellular staining for IFN- $\gamma$ or perforin was performed according to the BD Bioscience intracellular staining protocol and as described in (18). Briefly, surface staining was achieved with antiTCR $\delta$-chain Ab (TcR1-N24, GB21A; VMRD), followed by $\mathrm{PE}$ anti-mouse IgG2b (clone RMG2b-1, BioLegend, USA). The Cytofix/Cytoperm kit (BD Bioscience, USA) was used for cell fixation and permeabilization. Cytokines were labelled with FITC conjugated antibodies as follows: IFN $\gamma$ (clone CC302, AbSerotec, presently, Bio-Rad), IL-8 (Clone 8M6, detected with anti-mouse IgG2a-FITC, clone 6344, AbDSerotec), GM-CSF (clone CC305, detected with anti-mouse IgG2a-FITC, clone 6344, AbDSerotec). Surface expression of CD2, CD25, CD44, CD62L, CD335 and MHC II were assessed with anti-CD2-FITC (clone CC42, AbDSerotec), anti-CD25-FITC (clone IL-A111, AbDSerotec), anti-CD44-FITC (clone IL-A118, AbDSerotec), anti-CD62L-FITC (clone CC32, AbDSerotec), anti-CD335 (clone AKS1, detected with anti-mouse IgG2a-FITC, clone 6344, AbDSerotec) and ant-MHC II-FITC (clone IL-A21, AbDSerotec). Positive control cells were stimulated with poke weed mitogen (PWM). Cells assessed for CD335 and MHC II were compare to positive controls stimulated with LPS. One hundred thousand events were acquired in a FACSCalibur flow cytometer and analysed in CellQuest (BD Biosciences, USA).

Cytotoxicity. The principle behind this cytotoxicity assay is essentially that described in (19). Briefly, K562-GFP cells were infected with BCov at an MOI of 5 for $2 \mathrm{~h}$. Later, cells were thoroughly washed in RPMI-1640 and incubated with p102 or p156-stimulated $\gamma \delta$ T cells previously isolated from PBMCs for $4 \mathrm{~h}$ in RPMI-1640. Finally, 7-amino actinomycin D (7-AAD, live/dead discriminating dye; BD Biosciences) was added and incubated for 10 minutes. Staining with 7-AAD or lack thereof was assessed in FACSCalibur. Dead cells, i.e., those that spontaneously die or are killed by $\gamma \delta$ T cells are double stained (GFP/7-AAD), while live cells are GFP positive. One hundred thousand events were acquired in a FACSCalibur flow cytometer and analysed in CellQuest (BD Biosciences, USA).

Statistical analysis by Kruskal-Willis ANOVA was used in SIGMAPLOT 11.

\section{Results and discussion}

Out of the 4 cytokines used to assess the proliferative capacity of purified $\gamma \delta$ T cells, only IL-2 and hIL-15 stimulated the cells optimally at $48 \mathrm{~h}$ (Fig 2.) and were therefore further used to prepare various proliferative clones for generation of $\gamma$ and $\delta$ chains. Spectratypes from different clones (Fig. 3) were not highly varied, particularly for the $V \delta$ chains. Initially, 3 clones were selected for the sequencing of the CDR3 region, but these turned out to be very similar (Tab. 2) and therefore were regarded as a single clone. This suggested that stimulation with IL-2 and hIL-15 may have selected only this particular clone. Therefore, the cytokine environment may select for dominating clones bearing only a particular TCR. Future studies will assess more proliferative clones to increase chances of detecting diverse TCRs. The use of antigens for stimulation, in addition to cytokines, may also help to increase the diversity of $\gamma \delta \mathrm{T}$ cell clones for definition of ligands. Following infection of BTI-TN-5B1-4 cells with the baculovirus containing the sTCR supernatants showed production of a s $\gamma \delta$ TCR marked at $79 \mathrm{kDa}$ detected with anti- $\gamma \delta$ TCR antibody from VMRD (USA, unfortunately no longer available) (Fig. 4). A preparative non-denaturing PAGE was performed, and the soluble receptors were cut out of the polyacrylamide and eluted as described earlier and then used to detect $\mathrm{BCov}$ antigens in supernatants prepared from HRT-18 cells previously infected with BCov. The immunoblot technique adapted to detect the viral ligands showed that at least two proteins or protein fragments could bind to $\mathrm{s} \gamma \delta \mathrm{TCR}$ (Fig. 5). The predicted molecular weight of the bound viral ligands was found to be between 156 $\mathrm{kDa}$ and $102 \mathrm{kDa}$. It is likely that the high molecular weight band (approximately $150 \mathrm{kDa}$ ) corresponds to the molecular weight of the spike glycoprotein of

Tab. 2. Monoclonal CDR3 sequence (all clones have identical sequence for both $\gamma$ and $\delta$ chains)

\begin{tabular}{|c|l|c|}
\hline Clone name & V $\gamma$ CDR3 aa sequence & V $\delta$ CDR3 aa sequence \\
\hline CloneD1 & SGASAGYEQY & PGFTRGL/10 \\
CloneD2 & SGASAGYEQY & PGFTRGL/10 \\
\hline CloneD3 & SGASAGYEQY & PGFTRGL/10 \\
\hline
\end{tabular}




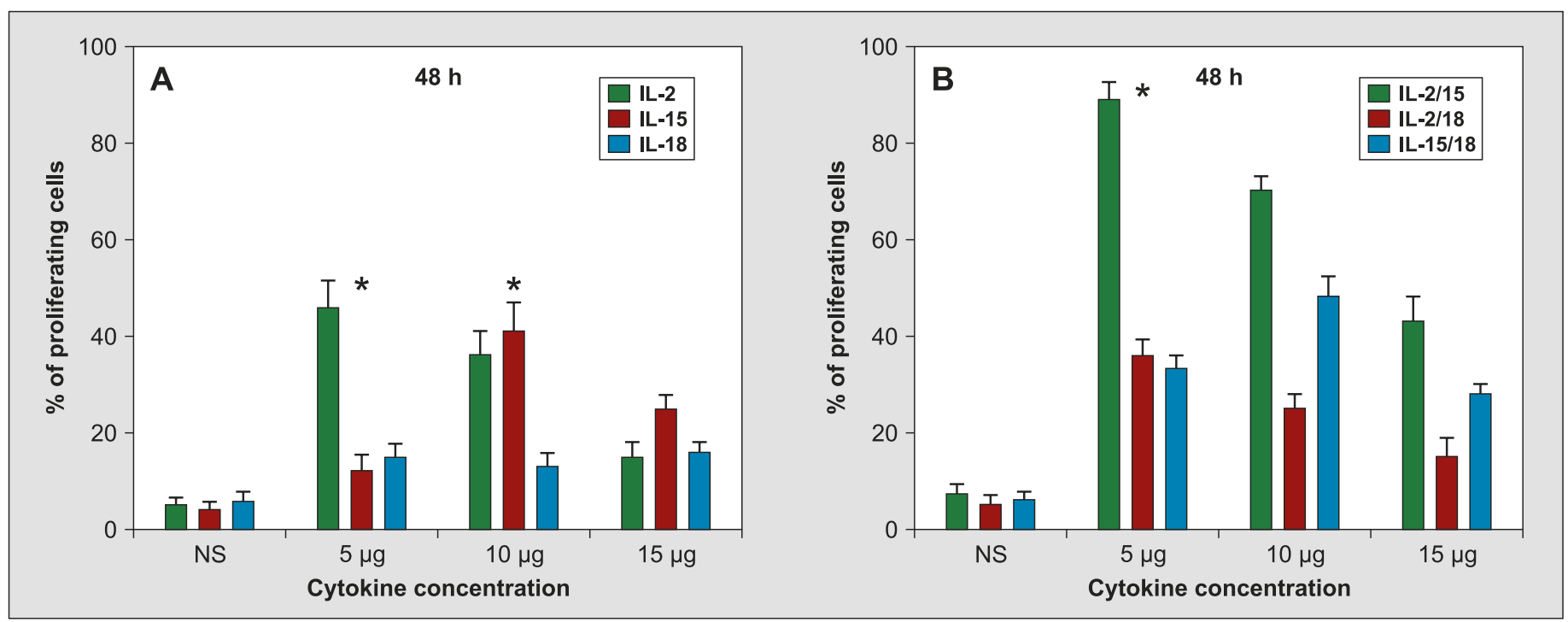

Fig. 2. Proliferation levels of WC1+ cells at concentrations of all cytokines used to select $\gamma \delta$ T cells for cloning

Explanations: Proliferation assay was performed with the MTT test. (A) individual cytokines; (B) combined cytokines. $\gamma \delta \mathrm{T}$ cells were isolated from blood samples by the MACS technique. Purity of cells was at least 95\%. Cells were later cultured in RPMI-1640 supplemented with $10 \%$ fetal bovine serum and cytokines investigated. ${ }^{*}=p \leq 0.05$ in comparison to NS (non-stimulated cells). Results shown are averages of 3 individual experiments, and values shown are percentages \pm SD.

BCov as reported by Abraham et al. (1). Because the BCov spike glycoprotein is made up of two subunits, it is possible that the second band, close to $102 \mathrm{kDa}$, is a subunit of the spike glycoprotein. Precise identification would be possible once protein sequencing has been carried out. Although the s $\gamma \delta$ TCR bound to the 2 proteins, the binding appears weak, which could possibly be attributed to dissociation of the chains under such conditions or a low affinity of the s $\gamma \delta T C R$ to ligand. However, attempts to use the Biacore labelfree surface plasmon resonance for affinity binding
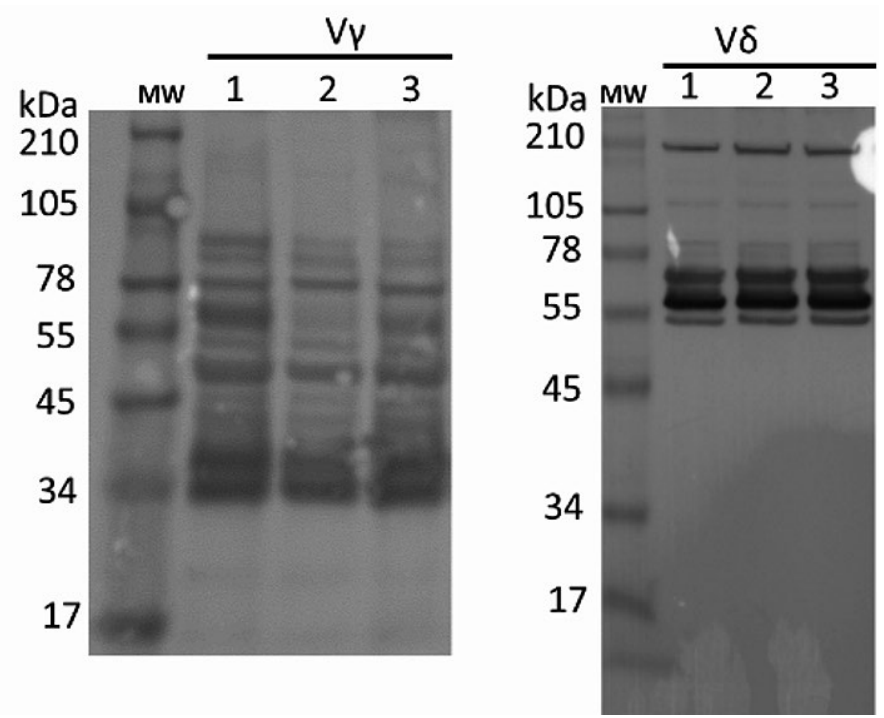

Fig. 3. CDR3 size spectratyping of $\gamma \delta$ T cells derived from IL-2/IL-15 culture in the presence of $\mathrm{BCV}$ antigens

Explanations: Total RNA was isolated from $\gamma \delta$ T cells, reverse-transcribed into cDNA, and amplified with specific primers. PCR products were run on a denaturing polyacrylamide gel and analysed with a Kodak In-vivo Imaging System FX fluorescence image analyser. Spectratypes representing oligoclonal expansion of $\mathrm{V} \gamma(\mathrm{A})$ and $\mathrm{V} \delta$ (B) cDNA for CloneD1, CloneD2 and CloneD3, $1,2,3$ respectively. assays did not yield acceptable results. The predicted protein bands from viral lysates were cut out of the polyacrylamide gel and eluted and after renaturing in $6 \mathrm{M}$ Guanidine- $\mathrm{HCl}$ solution, they were used to investigate the stimulatory properties on $\gamma \delta \mathrm{T}$ cells isolated from bovine PBMCs. For the purpose of this study, we named these 2 proteins as p156 and p102. First, we tested their capacity to stimulate cytokine production in

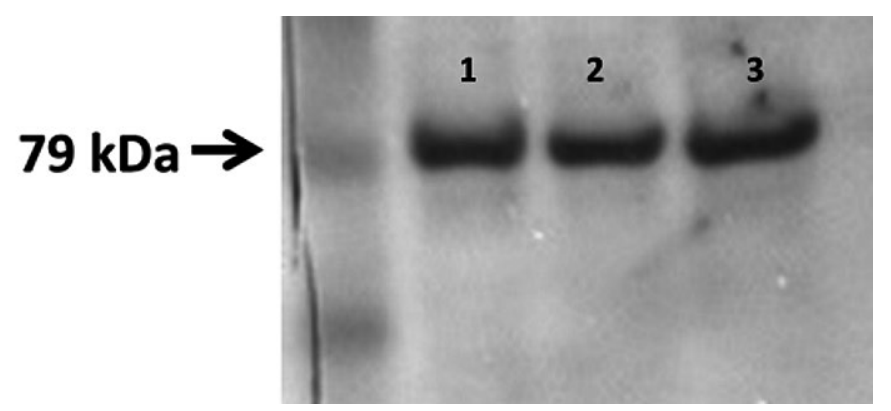

Fig. 4. Western blot image of supernatants from BTI-TN5B1-4 cells infected with the baculovirus vectors encoding Explanations: Clones 1, 2 or 3 cDNA, respectively for $s \gamma \delta T C R$. Electrophoresis, electrotransfer and protein detection were performed by standard procedures.

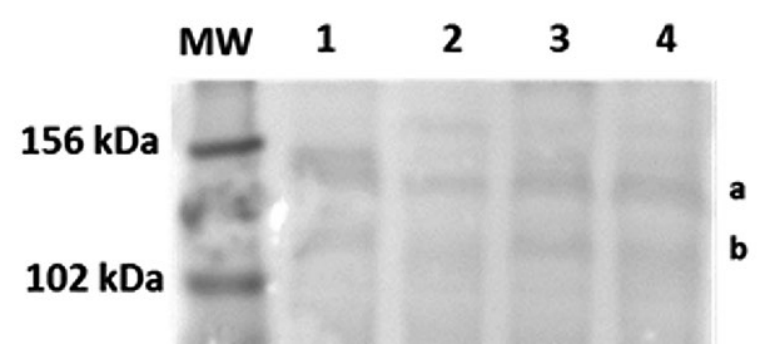

Fig. 5. BCV proteins detected in the supernatants of HRT-18 cells infected with bovine coronavirus by binding to $s \gamma \delta \mathrm{TCR}$ Explanations: An adapted immunoblotting technique was used to detect the viral proteins. (a) approximately $150 \mathrm{kDa}$, (b) approximately $110 \mathrm{kDa}$. 


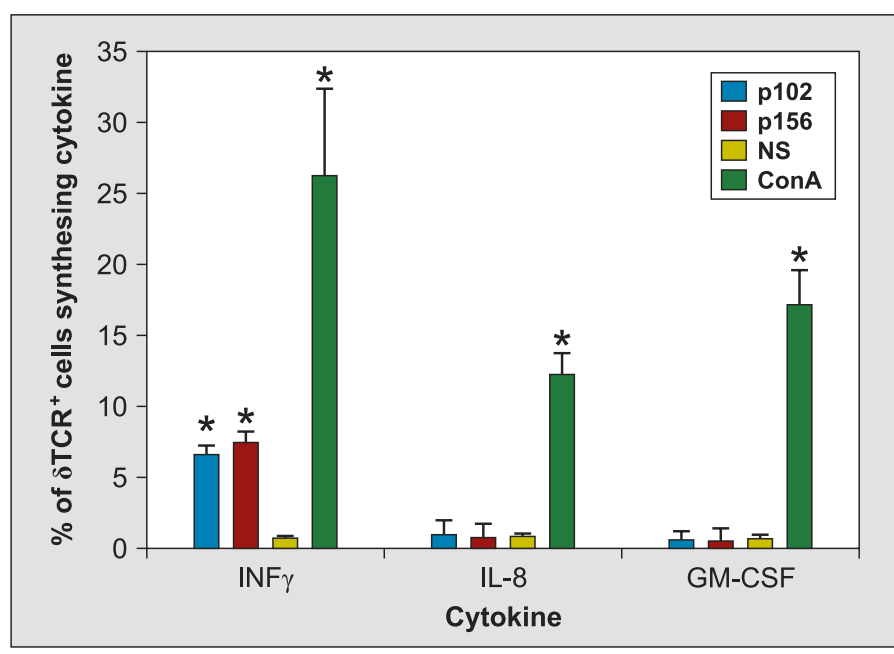

Fig. 6. Synthesis of selected cytokines by $\delta \mathrm{TCR}+\gamma \delta \mathrm{T}$ cells incubated with the $\mathrm{p} 156$ and $\mathrm{p} 102$ protein antigens isolated from HRT-18 cells infected with bovine coronavirus

Explanations: Cells were first sorted on $\mathrm{WC} 1$ and then investigated for cytokine synthesis by intracellular staining of $\delta \mathrm{TCR}+$ cells and flow cytometry. Data were plotted for $\delta \mathrm{TCR}+$ cells. ConA (concanavalin A) was included as a positive control. The figure represents average data sets from at least 3 separate experiments. $*=\mathrm{p} \leq 0.05$ compared to NS (non-stimulated cells) $; *=\mathrm{p} \leq 0.05$ compared to NS. Results are given as percentages \pm SD.

bovine $\mathrm{WC}^{+} \gamma \delta \mathrm{T}$ cells isolated from PBMCs. Many cytokines have been reported to be produced by bovine $\gamma \delta$ T cells stimulated by various ligands, e.g., bacterial ligands (20). We assessed IFN $\gamma$, IL-8 and GM-CSF to check whether viral ligands can lead to such reactivity. Figure 6 shows that both p156 and p102 were capable of inducing production of IFN $\gamma$ at a statistically significant level $(p \leq 0.05)$ and not IL-8 or GM-CSF (Fig. 6). This result shows that $\mathrm{WC1}^{+}$cells can directly respond to viral ligands. Others have shown responsiveness of $\mathrm{WCl}^{+}$cells to other antigens, such as Mycobacterium avium subspecies paratuberculosis, resulting in IFN $\gamma$ production (3). When surface marker expression had been analysed, it turned out that only MHC class II was expressed at least 4 -fold higher $(\mathrm{p} \leq 0.05)$ in the presence of p156 (Fig. 7). Although markers such as CD2, CD25, CD44, CD62L and CD335 were expressed at significant levels, only a single-fold difference could be observed. Indeed, as reported by Maślanka et al. (10), no correlation could be seen between CD25 expression and the production of IFN $\gamma$ in $\mathrm{WC}^{+} \gamma \delta \mathrm{T}$ cells, $17 \pm 1.2 \%$ (CD25) vs $6.5 \%$ (IFN $\gamma$ ). In another ruminant species antigenically stimulated with Mycobacterium avium subspecies paratuberculosis, a much larger increase in CD25 was observed (16). The choice of the surface markers examined in this study stems from our preliminary observations that cytokine- or mitogenstimulated cells increase the expression of CD2, CD25, CD44, CD62L, CD335 and MHC II, indicating their activation. Finally, as reported by others $(18), \mathrm{WC1}^{+}$ cells can kill targets in an antigen independent manner; therefore the capability of $\mathrm{WC}^{+} \gamma \delta \mathrm{T}$ cells to engage in cytotoxicity was assessed following in vitro stimulation

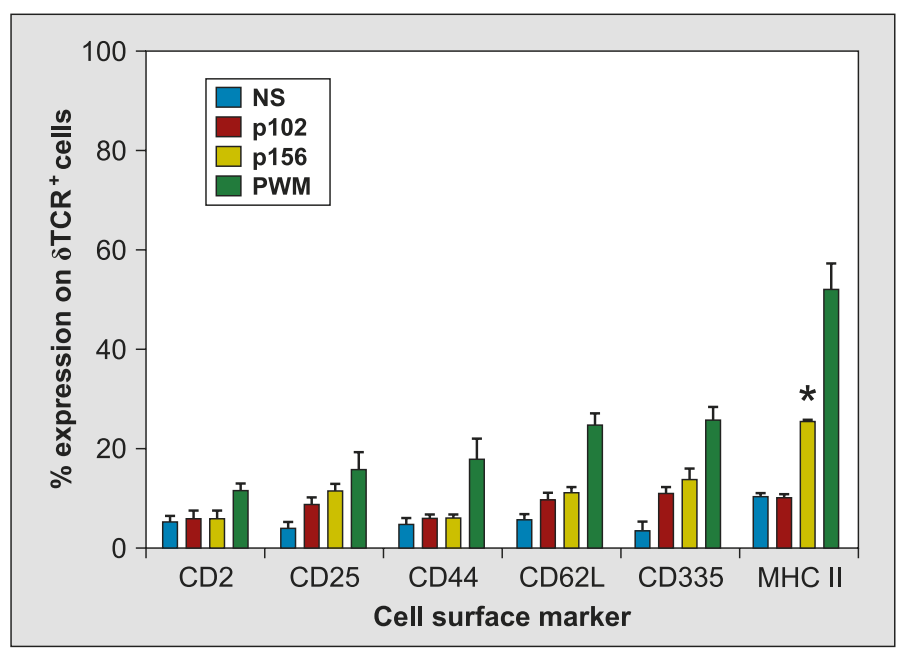

Fig. 7. Cell surface marker expression on $\delta \mathrm{TCR}+\gamma \delta \mathrm{T}$ cells treated with p102 or p156 identified through the designed STCR

Explanations: Flow cytometry was employed to measure cell surface expression. Pokeweed (PWM) mitogen was employed as a positive control. To assess CD335 and MHC II, LPS was used as a positive control (data not shown). The figure represents three averaged data sets from at least 4 separate experiments. $*=\mathrm{p} \leq 0.05$ compared to non-stimulated cells (NS) Results are given as percentages $\pm \mathrm{SD}$.

with p102 and p156 antigens. The cytotoxicity assay was performed essentially as described by Toka et al. (18). No reactivity against K562-GFP cells was evident besides that observed in the cells stimulated with cytokines (positive control) (Fig. 8); thus no cytotoxic effect was elicited by $\mathrm{WC}^{+} \gamma \delta \mathrm{T}$ cells stimulated with either $\mathrm{p} 102$ or $\mathrm{p} 156$. This result indicates that, although signs of stimulation in the form of IFN $\gamma$ production and increased MHC II expression were observed, $\mathrm{WC}^{+}$ $\gamma \delta$ T cells may require more or additional stimulus to engage in cytotoxicity of BCov-infected targets.

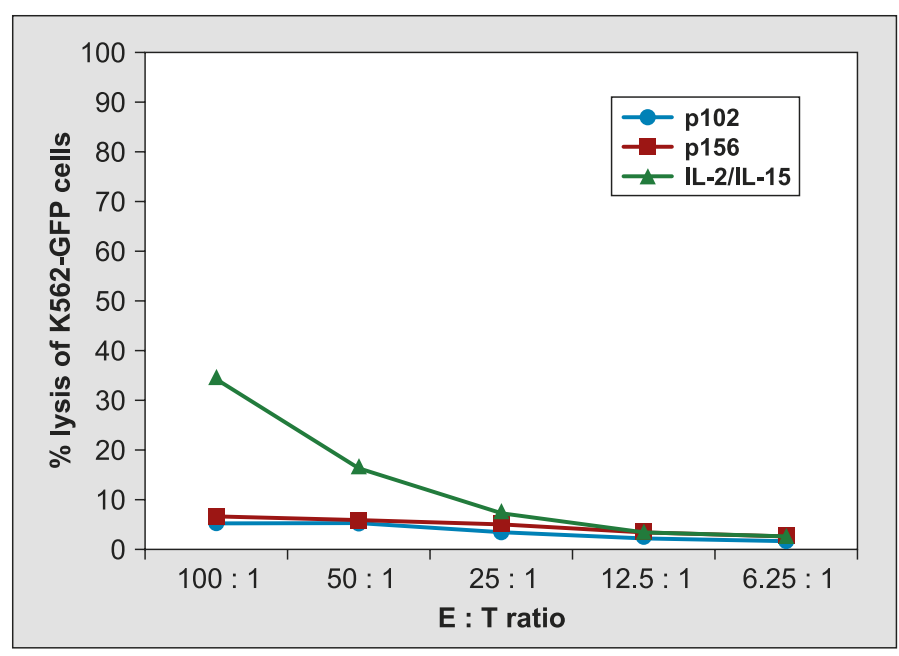

Fig. 8. A test of the cytotoxicity of $\gamma \delta \mathrm{T}$ cells treated with either p102 or p156 viral proteins identified through sTCR as stimulating antigens

Explanations: A flow cytometry-based assay was used to measure cytotoxicity against K562-GFP cells previously infected with BCov. The data represents a single experiment from at least 3 performed. 
Taken together, these results show a viable technique for constructing a sTCR for $\gamma \delta$ T cells, although only a single clone was derived. The method is rather laborious, and problems regarding the folding and association of chains in $T$. $n i$ cells were a disadvantage. This approach, however, made it possible to detect only the most abundant clone, omitting many of the subdominant ones. A different strategy was described by Steele et al. (17). Despite the difficulties, the results suggest that $\gamma \delta$ T cells can directly recognize antigens because the addition of protein antigen led to stimulation of the cells, culminating in IFN $\gamma$ production and increased expression of MHC class II on the cell surface. However, the biological significance of these results will be appreciated only by testing in in vivo experiments. Identification of ligands with the use of sTCRs can make it possible to discover antigens that might be useful in designing vaccines.

\section{References}

1. Abraham S., Kienzle T. E., Lapps W., Brian D. A.: Deduced sequence of the bovine coronavirus spike protein and identification of the internal proteolytic cleavage site. Virology 1990, 176, 296-301.

2. Aydintug M. K., Roark C. L., Yin X., Wands J. M., Born W. K., O'Brien R. L.: Detection of cell surface ligands for the gamma delta TCR using soluble TCRs. J. Immunol. 2004, 172, 4167-4175.

3. Baquero M. M., Plattner B. L.: Bovine WC1+ $\gamma \delta \mathrm{T}$ lymphocytes modify monocyte-derived macrophage responses during early Mycobacterium avium subspecies paratuberculosis infection. Vet. Immunol. Immunopathol. 2016, 170, 65-72.

4. Blumerman S. L., Herzig C. T. A., Rogers A. N., Telfer J. C., Baldwin C. L.: Differential TCR gene usage between $\mathrm{WC} 1-$ and $\mathrm{WC} 1+$ ruminant $\gamma \delta \mathrm{T}$ cell subpopulations including those responding to bacterial antigen. Immunogenetics 2006, 58, 680-692.

5. Chrobak A., Gmyrek G. B., Sozański R., Sieradzka U., Paprocka M., Gabryś M., Jerzak M.: The Influence of Extracellular Matrix Proteins on T-cell Proliferation and Apoptosis in Women with Endometriosis or Uterine Leiomyoma. A. J. Repro. Immunol. 2004, 51, 123-129.

6. Davodeau F., Houde I., Boulot G., Romagne F., Necker A., Canavo N., Peyrat M. A., Hallet M. M., Vie H., Jacques Y., et al.: Secretion of disulfide-linked human T-cell receptor gamma delta heterodimers. J. Biol. Chem. 1993, 268, 15455-15460.

7. Galluzzo S., Santini D., Vincenzi B., Caccamo N., Meraviglia F., Salerno A., Dieli $F$., Tonini $G$.: Immunomodulating role of bisphosphonates on human gamma delta T cells: an intriguing and promising aspect of their antitumour activity. Expert Opin. Ther. Targets 2007, 11, 941-954.
8. Green A. E., Lissina A., Hutchinson S. L., Hewitt R. E., Temple B., James D., Boulter J. M., Price D. A., Sewell A. K.: Recognition of nonpeptide antigens by human $\mathrm{V} \gamma 9 \mathrm{~V} \delta 2 \mathrm{~T}$ cells requires contact with cells of human origin. Clin. Exp. Immunol. 2004, 136, 472-482.

9. Hoek A., Rutten V. P. M. G., Kool J., Arkesteijn G. J. A., Bouwstra R. J., Van Rhijn I., Koets A. P.: Subpopulations of bovine WC1(+) $\gamma \delta \mathrm{T}$ cells rather than CD4(+)CD25(high)Foxp3(+) T cells act as immune regulatory cells ex vivo. Vet. Res. 2009, 40, 06.

10. Maślanka T., Jaroszewski J., Markiewicz W., Ziólkowski H., Barski D.: The presence of CD25 on bovine $\mathrm{WC} 1+\gamma \delta \mathrm{T}$ cells is positively correlated with their production of IL-10 and TGF- $\beta$, but not IFN- $\gamma$. Pol. J. Vet. Sci. 2012, 15, 11-20.

11. McGill J. L., Nonnecke B. J., Lippolis J. D., Reinhardt T. A., Sacco R. E.: Differential chemokine and cytokine production by neonatal bovine $\gamma \delta$ T-cell subsets in response to viral toll-like receptor agonists and in vivo respiratory syncytial virus infection. Immunology 2013, 139, 227-244.

12. Naoe M., Ogawa Y., Takeshita K., Morita J., Shichijo T., Fuji K., Fukagai T., Iwamoto S., Terao S.: Zoledronate stimulates gamma delta T cells in prostate cancer patients. Oncol. Res. 2010, 18, 493-501.

13. Palomares R. A., Sakamoto K., Walz H. L., Brock K. V., Hurley D. J.: Acute infection with bovine viral diarrhea virus of low or high virulence leads to depletion and redistribution of $\mathrm{WC} 1+\gamma \delta \mathrm{T}$ cells in lymphoid tissues of beef calves. Vet. Immunol. Immunopathol. 2015, 167, 190-195.

14. Peckham R. K., Brill R., Foster D. S., Bowen A. L., Leigh J. A., Coffey T. J. Flynn R. J.: Two distinct populations of Bovine IL-17(+) T-cells can be induced and $\mathrm{WC} 1(+) \mathrm{IL}-17(+) \gamma \delta$ T-cells are effective killers of protozoan parasites. Sci. Rep. 2014, 4, 5431.

15. Price S., Davies M., Villarreal-Ramos B., Hope J.: Differential distribution of $\mathrm{WC} 1+\gamma \delta \mathrm{TCR}+\mathrm{T}$ lymphocyte subsets within lymphoid tissues of the head and respiratory tract and effects of intranasal $\mathrm{M}$. bovis $\mathrm{BCG}$ vaccination. Vet. Immunol. Immunopathol. 2010, 136, 133-137.

16. Robinson M. W., O’Brien R., Mackintosh C. G., Griffin J. F. T.: Peripheral blood mononuclear cell population changes associated with age and Mycobacterium avium subspecies paratuberculosis infection in red deer (Cervus elaphus). Vet. Immunol. Immunopathol. 2010, 136, 211-218.

17. Steele C. R., Van Remoortere K. C., Hayday A. C.: Production of a soluble $\gamma \delta$ T-cell receptor to identify ligands for the murine intestinal intraepithelial $\gamma \delta$ T cell population. J. Chromatography B 2003, 786, 297-304.

18. Toka F. N., Kenney M. A., Golde W. T.: Rapid and Transient Activation of $\gamma \delta$ T Cells to IFN- $\gamma$ Production, NK Cell-Like Killing, and Antigen Processing during Acute Virus Infection. J. Immunol. 2011, 186, 4853-4861.

19. Toka F. N., Nfon C. K., Dawson H., Golde W. T.: Accessory-cell-mediated activation of porcine NK cells by toll-like receptor 7 (TLR7) and TLR8 agonists. Clin. Vaccine Immunol. 2009, 16, 866-878.

20. Wu P., Wu D., Ni C., Ye J., Chen W., Hu G., Wang Z., Wang C., Zhang Z., Xia W., Chen Z., Wang K., Zhang T., Xu J., Han Y., Zhang T., Wu X., Wang J., Gong W., Zheng S., Qiu F., Yan J., Huang J.: $\gamma \delta \mathrm{T} 17$ Cells Promote the Accumulation and Expansion of Myeloid-Derived Suppressor Cells in Human Colorectal Cancer. Immunity 2014, 40, 785-800.

Corresponding author: Felix N. Toka, DVM, PhD, DSc., DiplACVM, Department of Preclinical Sciences, Faculty of Veterinary Medicine, Warsaw University of Life Sciences, 02-787 Warsaw, Poland; e-mail: felix_toka@ sggw.pl 